\title{
Efecto del Láser de Baja Energía sobre la Expresión de GAP-43 (Growth Associated Protein 43) en Nervio Isquiático Lesionado de Rata
}

\author{
Low-Energy Laser Effect on Expression of GAP-43 \\ (Growth Associated Protein 43) in Rat Isquiatic Nerve Injury
}

"Fernando Matamala; ${ }^{* *}$ Marco Paredes \& ${ }^{* * * *}$ Ricardo Cornejo

MATAMALA, F.; PAREDES, M. \& CORNEJO, R. Efecto del láser de baja energía sobre la expresión de GAP-43 (growth associated protein 43) en nervio isquiático lesionado de rata. Int. J. Morphol., 28(3):815-821, 2010.

RESUMEN: El láser infrarrojo se ha utilizado con fines terapéuticos en artritis reumatoide, dolor músculo-esquelético, enfermedades dentales y óseas. A nivel de lesiones nerviosas, se ha utilizado como coadyuvante en tratamientos conservadores y quirúrgicos; sin embargo, no está claro aún cual es su participación en los mecanismos moleculares asociados al proceso regenerativo. Varios genes y sus productos de expresión relacionados con regeneración axonal, están siendo investigados, entre éstos, la proteína GAP-43 (growth associated protein 43) ha sido detectada en diferentes tipos de lesiones de nervios periféricos, donde su expresión aumenta notablemente durante el proceso de recuperación. Con el propósito de evaluar la expresión del transcrito de GAP-43 en nervio isquiático de rata lesionado y, posteriormente, irradiados con láser infrarrojo de $2 \mathrm{~J} / \mathrm{cm}^{2}$, se evaluó mediante RT- PCR en tiempo real la expresión del transcrito de GAP-43, en nervios lesionados tratados y no tratados. Los resultados indican que el nivel de expresión de GAP-43 en nervio tratado con láser de $2 \mathrm{~J} / \mathrm{cm}^{2}$, es mayor que en el nervio lesionado no tratado. También se observó un aumento de la expresión hasta dos semanas post-tratamiento. Estos resultados sugieren un efecto estimulante del láser infrarrojo de $2 \mathrm{~J} / \mathrm{cm}^{2}$ sobre la expresión de GAP-43, en nervio isquiático de rata lesionado por compresión, lo cual podría estar relacionado con un menor tiempo de regeneración del nervio.

PALABRAS CLAVE: Láser de baja energía, Nervio isquiático, GAP-43.

\section{INTRODUCTION}

Los primeros estudios realizados por Rochkind (1978), para evaluar el efecto del láser infrarrojo sobre nervio isquiático de rata dañado, demostraron que su aplicación fue capaz de estimular regeneración del nervio injuriado. Posteriormente este autor y sus colaboradores confirmaron el efecto benéfico del láser de baja energía sobre la regeneración de nervios periféricos y también de la formación de prolongaciones neuronales en el sistema nervioso central a nivel medular (Rochkind et al., 1987; 1988; Rochkind \& Ouaknine, 1992; Rochkind \& Alon, 2000). Estudios posteriores han demostrado que el efecto benéfico del láser parece estar relacionado con su capacidad para inducir generación de brotes neuríticos y proliferación de neuroglia como astrocitos y oligodendrogliocitos (Wollman \& Rochkind, 1998; Rochkind et al., 2001; Rossi et al., 2007). Cabe destacar que las repuestas neuronales a lesiones como axotomía o compresión axonal (neuropraxia) parecen estar relacionadas con la activación de diversos genes específicos cuyos productos de expresión son necesarios para el regeneración y remodelación del axón dañado (Lee et al., 2004; Qiu et al., 2005; Chierzi et al., 2005; Sung et al., 2006. Particularmente la proteína GAP-43 (growth associated protein 43) ha sido relacionada con el crecimiento del sistema nervioso durante el desarrollo embrionario y la regeneración de nervios en el adulto (Shin et al., 2003). Esta proteína aumenta notablemente en

\footnotetext{
* Unidad de Anatomía. Departamento de Ciencias Básicas. Facultad de Medicina. Universidad de La Frontera, Temuco, Chile.

** Laboratorio de Investigación en Biotecnología Animal. Departamento de Ciencias Básicas. Facultad de Medicina. Universidad de La Frontera, Chile.

*** Unidad de Biología. Departamento de Ciencias Básicas. Facultad de Medicina. Universidad de La Frontera, Temuco, Chile.
} 
tejido nervioso lesionado (Casoli et al., 2001; Ondarza et al., 2003; Rossi et al., 2007) y su nivel de expresión está corre-lacionado con la cantidad de brotes neuronales generados en el nervio en remodelación o regeneración (Van der Zee et al., 1989). Estudios basados en el análisis inmuno-histoquímico para GAP-43 en nervio ciático de rata, realizados por Shin et al. muestran que el nivel de expresión de esta proteína aumenta significativamente en nervios injuriados y tratados posteriormente con irradiación láser de baja energía. De este modo, el nivel de expresión de GAP-43 podría ser usado como un indicador de regeneración de un nervio dañado.

El propósito de este trabajo fue evaluar el efecto de la irradiación por láser de baja energía $\left(2 \mathrm{~J} / \mathrm{cm}^{2}\right)$ sobre la expresión del transcrito de GAP-43 en nervios isquiático de rata lesionados por compresión, comparando el nivel del transcrito mediante RT-PCR en tiempo real entre los nervios tratados y controles no tratados.

\section{MATERIAL Y MÉTODO}

Animales utilizados, generación de la injuria y tratamiento post-daño. Se utilizaron ratas Sprague Dawley de 250 gramos, mantenidas en el bioterio del Departamento de Ciencias Básicas de la Universidad de La Frontera. Previo a la intervención, para comprimir el nervio isquiático, las ratas fueron anestesiadas con ketamina $(9 \mathrm{mg} / 100 \mathrm{~g})$ y cilazina $(1.25 \mathrm{mg} / 100 \mathrm{~g})$. Posteriormente, se aisló quirúrgicamente el nervio isquiático, el cual fue comprimido por 3 minutos con una pinza de compresión controlada, aplicando una fuerza constante de $40 \mathrm{~N}$ en una sección del nervio de $10 \mathrm{~mm}$ sobre la bifurcación en nervio tibial y nervio peroneal común. Posteriormente, la herida fue desinfectada y suturada. Veinticuatro horas después de provocar la lesión, las ratas fueron irradiadas transcutáneamente en la zona correspondiente al recorrido del nervio, con un láser de arseniuro de galio (904 $\mathrm{nm} ; 10 \mathrm{~mW}$ ) regulado con una densidad de energía de $2 \mathrm{~J} /$ $\mathrm{cm}^{2}$. Se irradió por un tiempo de 10 minutos por día, durante 10 días consecutivos. Se utilizaron como control nervios de ratas lesionadas a las cuales no se les aplicó tratamiento de irradiación.

Un segundo control consistió en nervios de ratas no lesionadas pero tratadas con el mismo tipo de irradiación láser aplicado a las ratas lesionadas. Una vez concluidos los 10 días de tratamiento y 28 días post lesión, los animales fueron sacrificados con una sobredosis de anestésico para posteriormente, extraer los nervios. Luego de extraídos los nervios fueron congelados inmediatamente a $-80^{\circ} \mathrm{C}$, hasta el momento de la extracción de ARN.
Extracción de ARN y síntesis de ADN complementario. Se preparó $\mathrm{ADN}$ complementario (ADNc) a partir del ARN total extraído de los nervios isquiáticos lesionados e irradiados con láser infrarrojo y de los controles no tratados. El ARN fue extraido de acuerdo a metodología descrita por Chomczynski \& Sachi (1987), con leves modificaciones. La calidad del ARN obtenido se evaluó por electroforesis en gel de agarosa, en condiciones desnaturantes y su concentración se determinó mediante espectrofotometría, utilizando la ecuación [RNA] = OD260 x FD x $40 \mu \mathrm{g} / \mu \mathrm{l}$, donde FD es el factor de dilución utilizado y OD260 la absorbancia medida a $260 \mathrm{~nm}$. Mientras que la pureza se evaluó mediante la razón de absorbancia OD260/OD280, considerando valores de 1,8 a 2 como buena estimación de pureza. Para preparar ADNc mediante transcripción reversa, se utilizó 1 mg de RNA total, el cual se mezcló con tampón de transcripción consistente en: Tris- $\mathrm{HCl} 50 \mathrm{mM}$ pH 8,3 KCl $75 \mathrm{mM}, \mathrm{MgCl}_{2} 3 \mathrm{mM}$, dNTPs 10 mM, DTT 10 mM agregando, además, $40 \mathrm{U}$ de inhibidor de RNasa, oligo-dT20-tag 100 nM y 200 unidades de transcriptasa reversa. La mezcla de reacción se incubó a $37^{\circ} \mathrm{C}$ por 60 minutos. Luego se calentó a $70^{\circ} \mathrm{C}$ por 20 minutos para inactivar la transcriptasa. Finalmente, el ADNc fue utilizado de inmediato o mantenido a $20^{\circ} \mathrm{C}$ hasta su utilización.

Análisis de expresión del transcrito de GAP-43 por RT-PCR en tiempo real. El nivel de expresión del transcrito de GAP-43 se evaluó por medio de RT-PCR en tiempo real, utilizando el método "Comparative $\mathrm{Ct}$ " $(\Delta \mathrm{Ct})$ implementado en el sistema de amplificación en tiempo real "Step One" de Applied Biosystem. La cuantificación relativa expresa el número de veces (RQ) del nivel de expresión del gen estudiado en el tejido "problema", respecto al tejido control. Los resultados se obtuvieron por medio del cálculo que comprende la normalización de los valores de $\mathrm{Ct}$ obtenidos, respecto a un gen endógeno de expresión constante, que en este caso fue $ß$-actina, seguido del cálculo del $\Delta$ Ct y RQ (Fig. 1).

Condiciones de amplificación en Tiempo Real. Se utilizó ADNc como templado para la detección y amplificación específica del transcrito de GAP-43 y de $\beta$ actina, cuya expresión constitutiva fue utilizado como control endógeno referencial para la cuantificación relativa de GAP-43. El ADNc $(1 \mathrm{ml})$ se mezcló con $10 \mathrm{ml}$ del tampón de reacción, provisto en el kit "Power SYBR Green PCR Master Mix 2X" (Applied Biosystem, USA), $7 \mathrm{ml}$ de agua ultrapura $1 \mathrm{ml}$ del partidor GAP-43S: TGCTGTGCTGTATGAGAAGAACC (10 mM), $1 \mathrm{ml}$ del partidor GAP-43A: GGCAACGTGGAAAGCCGTTTCTTAAAG (10 $\mathrm{mM})$, para completar un volumen de reacción final de $20 \mathrm{ml}$. 
El ADNc de B-actina se amplificó mezclando $1 \mu \mathrm{l}$ de ADNc con $10 \mathrm{ml}$ del tampón de reacción "Power SYBR Green PCR Master Mix 2X" (Applied Biosystem), $1 \mathrm{ml}$ del partidor RBS: CATGTGCAAGGCCGGCTTCG $(10 \mathrm{mM})$, $1 \mathrm{ml}$ del partidor RBA: GTAGCAGGAGAAGTTGTTGG. (10 $\mathrm{mM}$ ) y $7 \mathrm{ml}$ de agua ultrapura completando un volumen de reacción final de $20 \mathrm{ml}$.

Las condiciones térmicas de amplificación para ambos genes fueron similares y se programaron con un ciclo inicial de 10 minutos a $95^{\circ} \mathrm{C}$, seguido por 45 ciclos compuestos cada uno por una denaturación a $95^{\circ} \mathrm{C}$ por 20 seg., hibridación a $60^{\circ} \mathrm{C}$ por 20 seg. y extensión a $72^{\circ} \mathrm{C}$ por $20 \mathrm{seg}$. Al término de los 45 ciclos de amplificación, se programó un ciclo para el análisis de las temperaturas de fusión (melting) de los productos de amplificación, lo cual permitió evaluar la especificidad de la reacción de amplificación y descartar de este modo el efecto de amplicones no específicos sobre la cuantificación de los templados.

Análisis estadístico. La significancia estadística de las diferencias entre los tratamientos experimentales y el control negativo, se evaluó con ANOVA y la prueba de comparación múltiple de Dunnett`s al nivel de 5\%, utilizando el programa Prisma 5.0

\section{Normalización}

\section{$\mathrm{C}_{\mathrm{t}}$ Gap $43-\mathrm{C}_{\mathrm{t}} \beta$ actina $=\Delta \mathrm{C}_{\mathrm{t}} 1$ \\ Muestra problema Control endógeno

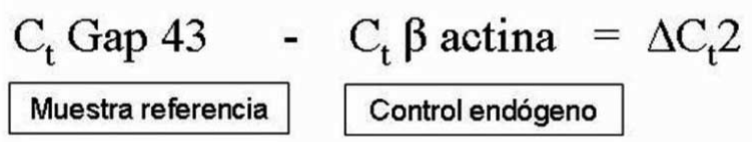

II. Obtención del RQ (Unidades de expresión relativa)

$$
\begin{aligned}
\Delta \mathrm{C}_{\mathrm{t}} 1-\Delta \mathrm{C}_{\mathrm{t}} 2 & =\Delta \Delta \mathrm{Ct} \\
2^{-\Delta \Delta \mathrm{Ct}} & =\mathrm{RQ}
\end{aligned}
$$

Fig. 1. Sistema de cuantificación relativa utilizada para evaluar el nivel de expresión de GAP 43 en nervio isquiático de rata. La cuantificación relativa se expresa como el "número de veces" (RQ) que el transcrito se expresa en relación a la muestra de referencia utilizada como control. Se utilizó el nivel de expresión de B-actina como gen referencial endógeno para normalizar el nivel de expresión de Gap43 tanto, en la muestra de referencia como en la muestra problema. Los valores de Ct (ciclo umbral) fueron obtenidos mediante RT-PCR en tiempo real con el sistema "StepOne" (Applied Biosystems).

\section{RESULTADOS}

Para asegurar que la evaluación cuantitativa del nivel de transcritos fuese consistente y confiable, se determinaron inicialmente diversos parámetros tales como la eficiencia, reproducibilidad y especificidad de la reacción de amplificación en tiempo real. Los resultados indicaron una eficiencia similar para el gen endógeno ( $(3-$ actina) y el gen problema (GAP-43). Además de la eficiencia, se calculó la reproducibilidad de la amplificación en tiempo real evaluando el coeficiente de variación de los ciclos umbrales entre amplificaciones independientes para el mismo tipo de muestra. De esta manera, el coeficiente de variación se determinó dividiendo la desviación estándar por la media aritmética de los valores de Ct obtenidos entre amplificaciones independientes de la misma muestra. La variabilidad entre ensayos independientes se determinó a partir de los valores de $\mathrm{Ct}$ del gen endógeno y gen problema. La diferencia absoluta en los valores de $\mathrm{Ct}$ fue inferior a 0,57 y 0,98 ciclos para cada amplificación independiente de ß-actina y GAP43 (Fig. 2).

Para determinar la especificidad de las amplificaciones, se analizó la curva de desnaturalización del producto de reacción de los estándares y las muestras a cuantificar. La curva de desnaturalización es generada por el software del termociclador (Step One; Applied Biosystems) el cual se programó para que, una vez concluido los ciclos de amplificación la temperatura, se eleve lentamente hasta $\operatorname{los} 95^{\circ} \mathrm{C}$. En nuestros resultados se obtuvo una única temperatura de desnaturalización para cada producto de amplificación, lo cual indica especificidad del producto amplificado (Fig. 2).

Los resultados de cuantificación relativa del transcrito de GAP-43, indican que los nervios lesionados por compresión presentan mayor nivel de expresión que el nervio control no lesionado (Fig. 3). Específicamente, el nervio lesionado y no tratado (ISQ 2) presenta aproximadamente 50 veces más expresión de GAP-43 que el nervio control no lesionado y no tratado (ISQ1). El nivel de expresión de GAP-43, se incrementa en el nervio lesionado e irradiado con láser de baja energía respecto al nervio control (ISQ1) en aproximadamente 150 veces (Fig. 3). El nervio no lesionado e irradiado aumento la expresión de Gap-43 más de 100 veces respecto al control ISQ1 (Fig. 3). Entre el nervio lesionado no tratado con láser y el nervio lesionado e irradiado se aprecia una diferencia de expresión significativa a favor de este último (Fig. 3). 


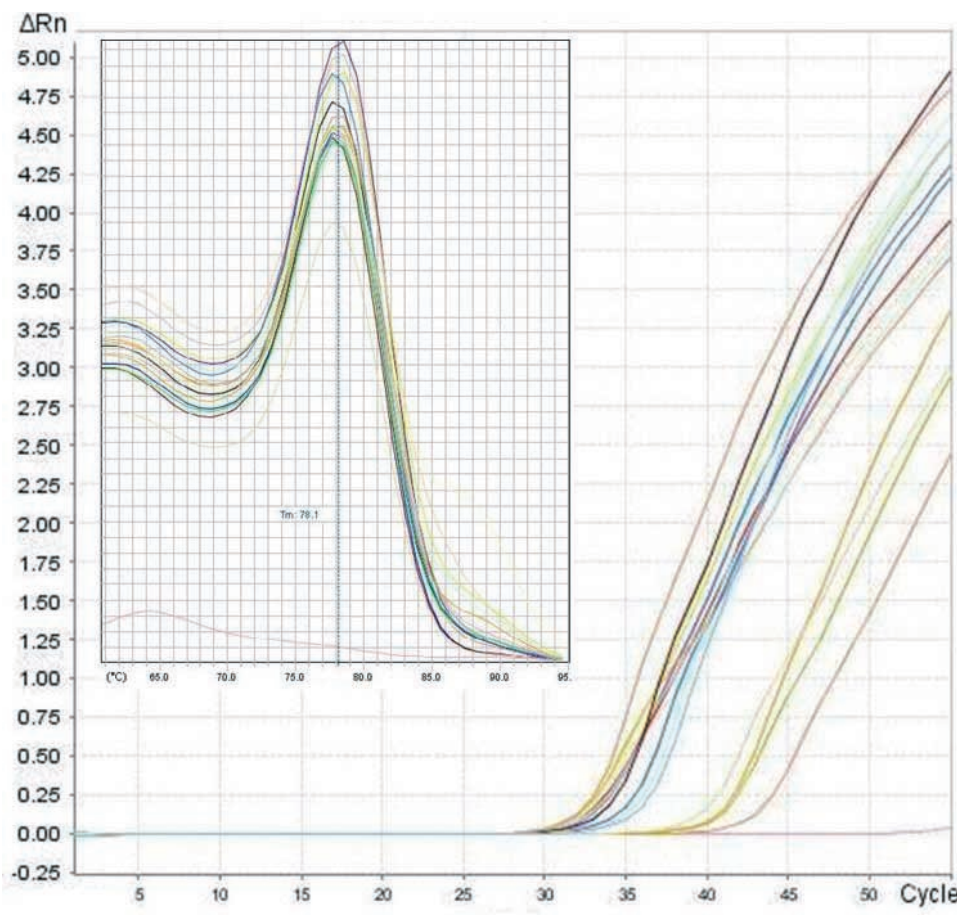

Fig. 2. Curvas de amplificación (recuadro exterior) y de fusión (recuadro interior) de los amplicones de GAP-43, obtenidos por RT-PCR en tiempo real, mediante el sistema StepOne (Applied Biosystems).

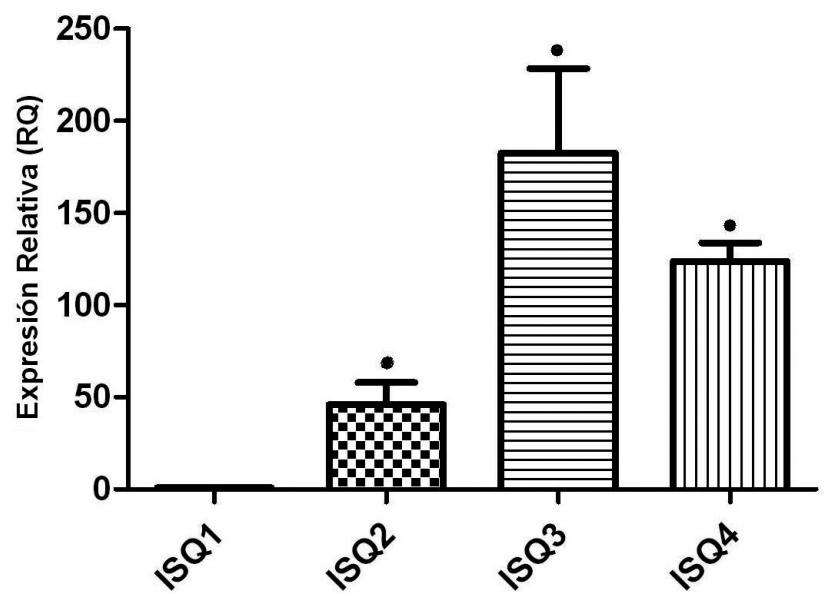

Fig. 3. Comparación del nivel expresión relativa del gen Gap-43 en nervio isquiático de rata sometido en vivo a lesión por compresión y posterior irradiación con láser de baja energía (ISQ3) y diversos controles experimentales. (ISQ1: Nervio sin lesión, no irradiado; ISQ2: Nervio con lesión, no irradiado; ISQ4: Nervio sin lesión, irradiado). Se determinó la expresión del transcrito de GAP43, utilizando unidades relativas definidas como "nuúmero de veces" (RQ) respecto a la expresión definida para el control de referencia (ISQ1) cuyo valor es 1 . El gráfico representa los valores medios \pm la desviación estándar, obtenidos en cuatro ensayos independientes por duplicado. La significancia estadística de las diferencias de los valores de expresión entre el control ISQ1 y los otros resultados, se evaluó con ANOVA y la prueba de comparación múltiple de Dunnett`s mediante el programa Prisma $5.0(\bullet p<0,05)$.

\section{DISCUSIÓN}

El efecto regenerativo de la irradiación con láser de baja energía en nervios periféricos dañados fue reportado inicialmente por Rochkind. Posteriormente numerosos estudios han confirmado el efecto benéfico del láser en la regeneración de diversos tipos de nervios periféricos lesionados (Rochkind et al., 1986; 1987; Anders et al; 1993; Shin et al.; Belchior et al., 2009). Sin embargo, los complejos mecanismos moleculares y celulares asociados al efecto regenerativo del láser recién comienzan a entenderse debido a que la regeneración axonal requiere cambios en el patrón de expresión molecular espacial y temporal, tanto en la neurona injuriada como también en células de Schwann, macrófagos y matriz extracelular asociadas al sitio de la lesión (Dubey et al., 1999; Valero-Cabré et al., 2004; Luk et al., 2003). Entre los diversos factores moleculares asociados a la regeneración del nervio lesionado, la proteína GAP-43 parece tener un papel preponderante (Skene \& Virag, 1989; Strittmatter, 1992; Shin et al.). Esta proteína es expresada por neuronas en proceso de regeneración tanto a nivel del sistema nervioso periférico como central (Shin et al.; Casoli et al.; Ondarza et al.). De este modo Gap-43 puede ser considerada un buen marcador molecular de regeneración neuronal.

El objetivo de este trabajo fue evaluar comparativamente el nivel de expresión de GAP-43 a nivel de su transcrito en nervios isquiáticos lesionados y controles sanos. Los resultados obtenidos indicaron que el nivel de expresión de GAP-43 es elevado en nervio isquiático de rata irradiado con láser de arseniuro de galio (904 $\mathrm{nm}$ ) regulado a una densidad de energía de $2 \mathrm{~J} / \mathrm{cm}^{2}$. Tanto en el nervio lesionado e irradiado, como en el control no lesionado e irradiado el nivel de expresión de GAP43 fue significativamente mayor que en el nervio lesionado no irradiado. El elevado nivel de expresión del transcrito de GAP-43 en nervio isquiático lesionado y posteriormente irradiado coincide con los resultados obtenidos por Shin et al. utilizando análisis inmunohistoquímico para la detección de GAP-43 en nervio isquiático de rata irradiado con láser de $5 \mathrm{~mW}$ de potencia y $650 \mathrm{~nm}$ de longitud de onda. Cabe destacar que el papel GAP-43 en regeneración de nervios periféricos aún no está completamente entendido, pero diversos tipos de estudio han demostrado claramente la asociación de esta proteína con procesos neuroregenerativos. Por ejemplo, 
la sobre-expresión de GAP-43 en ratones transgénicos está asociada a la aparición de brotes neuríticos espontáneos en poblaciones neuronales del sistema nervioso central y periférico, como también en la promoción de reinervación colateral en uniones neuromusculares (Aigner et al., 1995; Caroni et al., 1997), específicamente la sobre- expresión de GAP-43 en células de Purkinje transfectadas genera novedosas capacidades regenerativas en sus axones, las que no son evidenciadas en células controles no transfectadas con el gen (Rossi et al., 1995, 2006), poniendo de manifiesto la estrecha relación entre la presencia de GAP-43 en los lugares neuronales donde se brotes. También se ha visto que axones lesionados de células de Purkinje transfectadas con GAP-43, son capaces de generar brotes neuríticos en un ambiente de sustancia blanca, la cual es un reconocido factor inhibidor de brotes (Buffo et al., 1997; Gianola \& Rossi, 2004). Los resultados de esta experiencia también confirman el aumento de expresión de GAP-43 en nervio lesionado respecto al control sano; sin embargo, es interesante señalar que, mediante los análisis por RT-PCR en tiempo real, se pudo evaluar el nivel del transcrito de GAP-43 en una sección del nervio completo, la cual incluye axones, células de Schwann, fibroblastos, fibrocitos y otras células, como macrófagos en los nervios lesionados. Existen evidencias de expresión no neuronal de GAP-43 en células de Schwann (Curtis et al., 1992; Scherer et al., 1994), por lo que los niveles de expresión del transcrito de GAP-43 mostrados en nuestros resultados podrían estar influenciados por la expresión en células de Schwann. Estudios futuros, mediante hibridación in situ e inmunohistoquímica, podrían aclaran la distribución celular del transcrito y proteína de GAP-43 en nervio isquiático.

Los resultados obtenidos indican que la irradiación con láser de baja energía induce aumento del ARNm de GAP-43 en el nervio no lesionado. Estos resultados su-

MATAMALA, F.; PAREDES, M. \& CORNEJO, R. Low-energy laser effect on expression of GAP-43 (growth associated protein 43) in rat isquiatic nerve injury. Int. J. Morphol., 28(3):815-821, 2010.

SUMMARY: The Low-energy laser has been used in therapy for rheumatoid arthritis, musculoskeletal pain, dental disease and bone disease. At the level of nerve injury has been used as an adjunct to conservative and surgical treatments is not clear yet although the molecular mechanisms associated with the regenerative process. Several genes and their expression products associated with axonal regeneration are being investigated between these the protein GAP-43 (Growth associated protein 43) has been detected in different types of peripheral nerve injuries, where its expression increases markedly during the recovery process. In order to evaluate the expression of GAP-43 transcript in rat injured sciatic nerve and then irradiated with infrared laser of $2 \mathrm{~J} / \mathrm{cm}^{2}$ was evaluated by RT-PCR in real time transcript expression of GAP-43 in nerve treated and untreated injuries. The results indicate that the expression level of GAP-43 in nerve treated with $2 \mathrm{~J} / \mathrm{cm}^{2}$ laser is higher than in untreated injured nerve him. There was also an increased expression up to two weeks posttreatment. These results suggest a stimulatory effect of $2 \mathrm{~J} / \mathrm{cm}^{2}$ infrared laser on the expression of GAP-43 in rat injured nerve compression, which could be related to a shorter time of regeneration of injured nerve. gieren un efecto fotoinductor del láser sobre la expresión de GAP-43 no sólo en el nervio dañado sino también en el nervio sano. Aún no existen evidencias que puedan explicar el efecto directo del láser sobre el nivel de trascripción génica de GAP-43; sin embargo, algunos estudios indican que el láser podría alterar el nivel del cal2004) lo cual podría inducir vías de señalización intracelular comprometidas en expresión génica de GAP-43.

El papel de GAP-43 en regeneración neuronal está bien establecido, sin embargo, el efecto del láser sobre la expresión de esta proteína aún requiere estudios que permitan explicar tanto los mecanismos inductores de su expresión como de su papel en la regeneración neuronal.

\section{CONCLUSION}

La aplicación trascutánea de irradiación láser de arseniuro de galio $(904 \mathrm{~nm} ; 10 \mathrm{~mW})$ con una densidad de energía de $2 \mathrm{~J} / \mathrm{cm}^{2}$ sobre nervio isquiático lesionado por compresión en rata Sprague Dawley, incrementa significativamente la expresión del transcrito de GAP-43 con respecto al control no tratado.

\section{AGRADECIMIENTOS}

Dirección de Investigación y Desarrollo de la UniA los ayudantes de investigación: Daniela Iturriaga Figueroa, Leonardo Lafuente Astroza y Frans Navarro Navarro. A los tesistas del Magister en Ciencias mención Morfología Manuel Maurelia Fuentes y Emilio Farfán Cabello.

KEY WORDS: Low-energy laser, Isquiatic nerve, GAP-43. 


\section{REFERENCIAS BIBLIOGRAFICAS}

Aigner, L.; Arber, S.; Kapfhammer, J. P.; Laux, T.; Schneider, C.; Botteri, F., Brenner, H. \& Caroni, P. Overexpression of the neural growth associated protein GAP-43 induces nerve sprouting in the adult nervous system of transgenic mice. Cell, 83:269-78, 1995.

Anders, J. J.; Borke, R. C.; Woolery S. K. \& Van de Merwe W. P. Low power laser irradiation alters the rate of regeneration of the rat facial nerve. Lasers Surg. Med., 13:72-82, 1993.

Belchior, A. C.; dos Reis, F. A.; Nicolau, R. A.; Silva, I. S.; Perreira, D. M. \& de Carvalho, P. de T. Influence of laser $(660 \mathrm{~nm})$ on functional recovery of the sciatic nerve in rats following crushing lesion. Lasers Med. Sci., 24:8939, 2009.

Buffo, A.; Holtmaat, A. J.; Savio, T.; Verbeek, S.; Oberdick, J.; Oestreicher, A. B.; Gispen, W. H., Verhaagen, J.; Rossi, F. \& Strata, P. Targeted overexpression of the neurite growth-associated protein B-50/GAP-43 in cerebellar Purkinje cells induces sprouting after axotomy but not axon regeneration into growth-permissive transplants. J. Neurosci., 17:877891, 1997.

Caroni, P.; Aigner, L. \& Schneider, C. Intrinsic neuronal determinants locally regulate extrasynaptic and synaptic growth at the adult neuromuscular junction. J. Cell. Biol., 136: 679-92, 1997.

Casoli, T.; Di Stefano, G.; Gracciotti, N.; Giovagnetti, S.; Fattoretti, P.; Solazzi, M. \& Bertoni-Freddari, C. Cellular distribution of GAP-43 mRNA in hippocampus and cerebellum of adult rat brain by in situ RT-PCR. J. Histochem. Cytochem., 49(9):1195-6, 2001.

Chierzi, S.; Ratto, G. M.; Verma, P.; \& Fawcett, J. W. The ability of axons to regenerate their growth cones depends on axonal type and age, and is regulated by calcium, cAMP and ERK. Eur. J. Neurosci. 21:2051-62, 2005.

Chomczynski, P. \& Sacchi, N. Single-step method of RNA isolation by acid guanidinium thiocyanate-phenolchloroform extraction. Anal. Biochem., 162:156-9, 1987.

Curtis, R.; Stewart, J. S; . Hall, S. M.; Wilkin, G. P.; Mirsky, R. \& Jessent, K. R. GAP-43 is expressed by nonmyelin-forming schwann cells of the peripheral nervous system. J. Cell Biol., 116:1455-64, 1992.
Dubey, N.; Letourneau, P. C. \& Tranquillo, R. T. Guided neurite elongation and Schwann cell invasion into magnetically aligned collagen in simulated peripheral nerve regeneration. Exp. Neurol., 158:338-50, 1999.

Gianola, S. \& Rossi, F. GAP-43 overexpression in adult mouse Purkinje cells overrides myelin-derived inhibition of neurite growth. Eur. J. Neurosci., 19:819-30, 2004.

Klebanov, G. I.; Kreinina, M. V.; Poltanov, E. A.; Khristoforova, T. V. \& Vladimirov, Y. A. Mechanism of therapeutics effect of low intensity infrared laser irradiation. Bull. Exp. Biol. Med., 131: 239-41, 2001.

Lee, N.; Neitzel, K. L.; Devlin, B. K.; \& MacLennan, A. J. STAT3 phosphorylation in injured axons before sensory and motor neuron nuclei: Potential role for STAT3 as a retrograde signaling transcription factor. J. Comp. Neurol., 474: 535-45, 2004.

Luk, H. W.; Noble, L. J. \& Werb, Z. Macrophages contribute to the maintenance of stable regenerating neurites following peripheral nerve injury. J. Neurosci. Res., 73: 644-58, 2003.

Ondarza, A. B.; Ye, Z. \& Hulsebosch, C. E. Direct evidence of primary afferent sprouting in distant segments following spinal cord injury in the rat: Colocalization of GAP-43 and CGRP. Exp. Neurol., 184:373-80, 2003.

Qiu, J.; Cafferty, W. B.; McMahon, S. B. \& Thompson, S. W. Conditioning injury induced spinal axon regeneration requires signal transducer and activator of transcription 3 activation. J. Neurosci., 25:1645-53, 2005.

Rochkind, S. Stimulation effect of laser energy on the regeneration of traumatically injured peripheral nerves. Morphogen. Regen., 83:25-7, 1978.

Rochkind, S. \& Alon, M. Microsurgical management of old injuries of the peripheral nerve and brachial plexus. $J$. Reconstr. Microsurg., 16:541-6, 2000.

Rochkind, S.; Barr-Nea, L.; Razon, N.; Bartal, A. \& Schwartz M. Stimulatory effect of He-Ne low dose laser on injured sciatic nerves of rats. Neurosurgery, 20:8437, 1987.

Rochkind, S.; Nissan, M.; Alon, M.; Shamir, M. \& Salame K. Effects of laser irradiation on the spinal cord for the 
MATAMALA, F.; PAREDES, M. \& CORNEJO, R. Efecto del láser de baja energía sobre la expresión de GAP-43 (growth associated protein 43) en nervio isquiático lesionado de rata. Int. J. Morphol., 28(3):815-821, 2010.

regeneration of crushed peripheral nerve in rats. Lasers Surg. Med., 28:216-9, 2001.

Rochkind, S.; Nissan, M.; Lubart, R.; Avram, J. \& Bartal, A. The in-vivo-nerve response to direct low-energy-laser irradiation. Acta Neurochir., 94:74-7, 1988.

Rochkind, S.; Nissan, M.; Razon, N.; Schwartz, M. \& Bartal A. Electrophysiological effect of HeNe laser on normal and injured sciatic nerve in the rat. Acta Neurochir., 83:125-30, 1986.

Rochkind, S. \& Ouaknine, G. E. New trend in neuroscience: lowpower laser effect on peripheral and central nervous system. Neurol. Res., 14:2-11, 1992

Rossi, F.; Gianola, S.; \& Corvetti L. Regulation of intrinsic neuronal properties for axon growth and regeneration. Prog. Neurobiol., 81:1-28, 2007.

Rossi, F.; Gianola, S. \& Corvetti, L. The strange case of Purkinje axon regeneration and plasticity. Cerebellum 5:174-82, 2006.

Rossi, F.; Jankovski, A. \& Sotelo, C. Differential regenerative response of Purkinje cell and inferior olivary axons confronted with embryonic grafts: Environmental cues versus intrinsic neuronal determinants. J. Comp. Neurol., 359:663-77, 1995.

Rummler, L. S.; Paul, T. D. \& Gupta R. The anatomy and biochemistry of myelin and myelination. Oper. Tech. Orthop., 14:146-52, 2004.

Scherer, S. S.; Xu, Y. T.; Roling, D.; Wrabetz, L.; Feltri, M. L. \& Kamholz, J. Expression of growth-associated protein-43 kD in Schwann cells is regulated by axonSchwann cell interactions and cAMP. J. Neurosci. Res., 38(5):575-89, 1994.

Shin, D. H,; Lee, E.; Hyun, J. K.; Lee, S. J.; Chang, Y. P. \& Kim, J. W. Growth-associated protein-43 is elevated in the injured rat sciatic nerve after low power laser irradiation. Neurosci. Lett., 344:71-4, 2003.

Skene, J. H. \& Virag, I. Posttranslational membrane attachment and dynamic fatty acylation of a neuronal growth cone protein, GAP- 43. J. Cell Biol., 108:61324, 1989.

Strittmatter, S. M. GAP-43 as a modulator of G protein transduction in the growth cone. Perspect. Dev. Neurobiol., 1:13-9, 1992.
Sung, W. J.; Chiu, D. T. \& Ambron, R. T. Activation and retrograde transport of protein kinase $G$ in rat nociceptive neurons after injury or inflammation. Neuroscience, 141:697-709, 2006.

Valero-Cabre', A. Peripheral and spinal motor reorganization after nerve injury and repair. J. Neurotrauma, 21:95-108, 2004.

Van der Zee, C. E.; Nielander, H. B.; Vos, J. P.; Lopes da Silva, S.; Verhaagen, J.; Oestreicher, A. B.; Schrama, L. H.; Schotman, P. \& Gispen, W.H. Expression of growth associated protein B-50 (GAP43) in dorsal root ganglia and sciatic nerve during regenerative sprouting. J. Neurosci., 9:3505-12, 1989.

Wollman, Y. \& Rochkind S. In vitro cellular processes sprouting in cortex microexplants of adult rat brains induced by low power laser irradiation. Neurol Res., 20:470-2, 1998.

Correspondencia para autor:

Marco Paredes

Laboratorio de Investigación en Biotecnología Animal

Departamento de Ciencias Básicas

Facultad de Medicina

Universidad de La Frontera

Temuco

CHILE

Telefono: 56-45-325217

E-mail: mparedes@ufro.cl

Recibido : 22-03-2010

Aceptado: 21-07-2010 
